\title{
Performance Evaluation of Two LMMSE Detectors in a MIMO-OFDM Hardware Testbed
}

\author{
Markus Myllylä and Markku Juntti \\ University of Oulu, Centre for Wireless Communications \\ P.O. Box 4500, FI-90014 University of Oulu, Finland \\ \{markus.myllyla, markku.juntti\}@ee.oulu.fi \\ Matti Limingoja and Aaron Byman \\ Elektrobit Ltd. \\ Tutkijantie 8, FI-90570 Oulu, Finland \\ \{matti.limingoja, aaron.byman\}@elektrobit.com \\ Joseph R. Cavallaro \\ Dept. of Electrical \& Computer Engineering \\ Rice University, Houston, TX 77251-1892, USA \\ cavallar@rice.edu
}

\begin{abstract}
The performance of two field programmable gate array (FPGA) implementations of a linear minimum mean square error (LMMSE) based detector is evaluated in realtime radio channels. Two square root free algorithms based on the $\mathrm{QR}$ decomposition (QRD) via Givens rotations, namely coordinate rotation digital computer (CORDIC) and squared Givens rotation (SGR) algorithms, are applied for the LMMSE detector implementation with pipelined systolic array architectures. The implementations are mapped to Elektrobit $2 \times 2$ multiple-input multiple-output orthogonal frequency division multiplexing (MIMO-OFDM) hardware testbed for 4G MIMO systems (EB4G). The presented measurement results are done with a Propsim C8 MIMO channel emulator and compared to the simulated results.
\end{abstract}

\section{INTRODUCTION}

Multiple-input multiple-output (MIMO) techniques in combination with orthogonal frequency division multiplexing (OFDM) technique (MIMO-OFDM) have been identified as a promising approach for high spectral efficiency wideband systems [1], [2]. The optimal detection method for coded systems would be the maximum a posteriori (MAP) detection. However, the computational complexity of optimal MAP detection is beyond the limit of most systems, and, thus, such an approach is not feasible. A suboptimal approach is to use suboptimal zero forcing ( $\mathrm{ZF}$ ) or minimum mean square error (MMSE) criterion based linear detectors [3].

Several approaches exist to solve the matrix inversion required by the LMMSE detector [4], [5]. In this paper, two square root free algorithms are considered for the implementation of a LMMSE detector. The algorithms, namely the coordinate rotation digital computation (CORDIC) [6] algorithm and the squared Givens rotation (SGR) [7] algorithm, are applied to compute the QR decomposition (QRD) via Givens rotations. Then the matrix inversion is obtained by using a triangular matrix inversion algorithm [8] or a back substitution algorithm [4]. Two detector architectures, based on these algorithms and systolic array structures [9], [10], are designed for a $2 \times 2$ MIMO-OFDM system and implemented in a field programmable gate array (FPGA) chip. The pipelined architectures are fast, parallel, and suitable for OFDM systems where the calculation of detector coefficients has to be done for multiple subcarriers in channel coherence time.

The FPGA implementations of the detectors are mapped to the Elektrobit OFDM testbed for 4G MIMO systems (EB4G). The performance of the algorithms is evaluated using the EB4G hardware testbed and a Propsim C8 MIMO channel emulator creating the four baseband channels in the $2 \times 2 \mathrm{MIMO}$ system in real-time. The performance results in different real time channels are presented and evaluated.

The paper is organized as follows. The system model is presented in Section II. The designed architectures are presented in Section III. The hardware implementation in FPGA is presented in Section IV. The performance results are presented in Section V. Summary and Conclusions are presented in Section VI.

\section{SYSTEM MODEL}

A MIMO-OFDM system is considered with two transmit antennas and two receive antennas as shown in Figure 1. The received signal can be expressed in terms of the code symbol interval as

$$
\mathbf{r}_{p}=\mathbf{H}_{p} \mathbf{x}_{p}+\boldsymbol{\eta}_{p}, \quad p=1,2, \ldots, P,
$$

where $P$ is the number of subcarriers and the received signal vector, the transmit symbol vector and the noise vector are defined in the frequency domain, respectively, as $\mathbf{r}_{p}=$ 


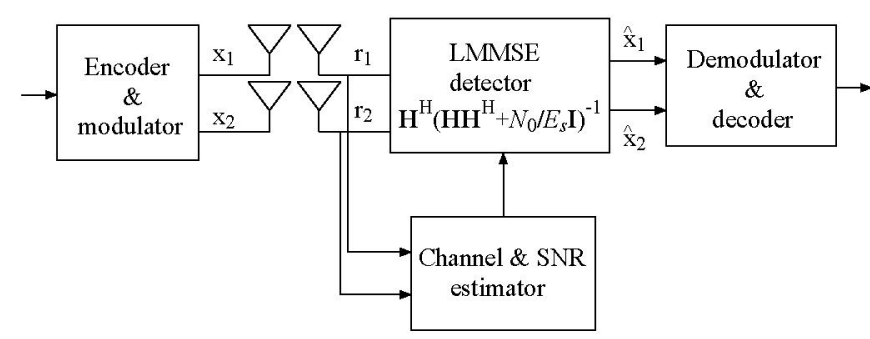

Fig. 1. Model of a MIMO system with two transmit and two receive antennas.

$\left[r_{p, 1}, r_{p, 2}\right]^{\mathrm{T}}, \mathbf{x}_{p}=\left[x_{p, 1}, x_{p, 2}\right]^{\mathrm{T}}, \boldsymbol{\eta}_{p}=\left[\eta_{p, 1}, \eta_{p, 2}\right]^{\mathrm{T}}$. The elements of $\boldsymbol{\eta}_{p}$ are independent and complex Gaussian with equal power real and imaginary parts, i.e., $\eta_{p} \sim \mathcal{C N}\left(\mathbf{0}, N_{0} \mathbf{I}_{2}\right)$ and represent the frequency domain thermal noise at the receiver. The channel matrix $\mathbf{H}_{p} \in \mathbb{C}^{2 \times 2}$ contains complex Gaussian fading coefficients with unit variance.

\section{A. LMMSE Detector}

The LMMSE based detector [3] minimizes the MSE between the transmitted signal vector $\mathbf{x}_{p}$ and the soft output vector of the LMMSE front end $\hat{\mathbf{x}}_{p}=\mathbf{W}_{p}^{\mathrm{H}} \mathbf{r}_{p}$. By using the well known Wiener solution [11], the LMMSE detector for MIMO-OFDM can be reduced to

$$
\mathbf{W}_{p}=\left(\mathbf{H}_{p} \mathbf{H}_{p}^{\mathrm{I}}+\frac{N_{0}}{E_{\mathrm{s}}} \mathbf{I}_{2}\right)^{-1} \mathbf{H}_{p},
$$

where we assume $\mathbf{R}_{x x}=E_{\mathrm{s}} \mathbf{I}_{2}$ and $\mathbf{R}_{\eta \eta}=N_{0} \mathbf{I}_{2}$.

The calculation of the LMMSE solution in (2) requires a matrix inversion operation which is computationally a very complex task. In this paper, two square root free methods based on QRD via Givens rotations are considered for calculation of the matrix inversion in (2):

- The CORDIC [6] + the back substitution [4] algorithms

- The SGR [7] + triangular matrix inversion [8] algorithms For more details, see [12].

\section{ARCHITECTURE}

The architectural design of matrix operations in LMMSE detectors are based on systolic array structures with communicating processing elements (PEs) [9], [10]. A simple and highly parallel triangular array architecture is applied for computing the QRD [9]. The architecture enables a simple data flow and achieves high throughput with pipelining. This is important in MIMO-OFDM system, where the detector coefficients are calculated separately for each subcarrier in the interval of the channel coherence time. Both the algorithm for inversion of a triangular matrix [8] and the back substitution algorithm [4] are implemented using a triangular array architecture.

The high level architecture of the LMMSE detector is presented in Figure 2. Computationally the most complex part of the detector is the coefficient calculation block, i.e., the calculation of (2). The CORDIC and SGR based architectures for $2 \times 2$ LMMSE detector coefficient matrix calculation are illustrated in Figure 3 and in Figure 4, respectively. The matrix to be inverted in (2) is formed in part $\mathrm{A} 1$. The matrix inversion

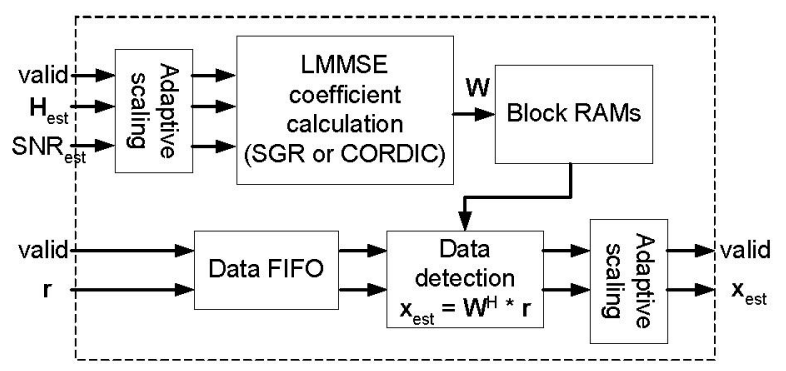

Fig. 2. The LMMSE detector high level architecture.

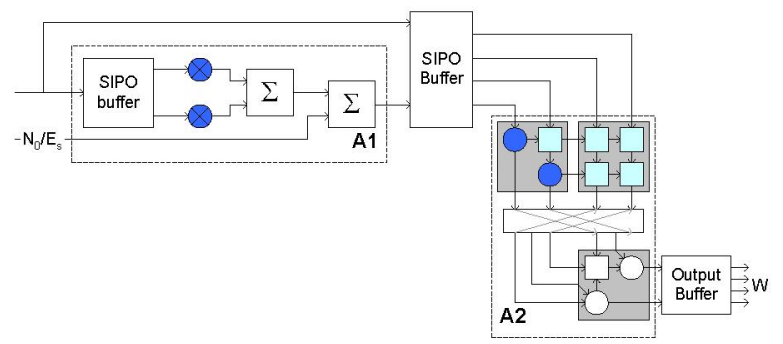

Fig. 3. The CORDIC based LMMSE detector architecture for $2 \times 2$ system.

is then calculated in part A2 which consists of two systolic arrays. The $\mathrm{QRD}$ of the matrix to be inverted is calculated using CORDIC or SGR algorithms in the upper systolic array. The lower systolic array applies the back substitution or the triangular matrix inversion algorithm. The matrix-matrix multiplication of the inverted matrix and the channel matrix is calculated in the part A3 in the SGR based architecture and in back substitution in part A2 in CORDIC based architecture. For more details of the architectures, see [12]. The architectures applied do not require much control logic and the mapping of data flow is relatively straightforward.

\section{IMPLEMENTATION}

The FPGA implementations of the detectors are mapped to the EB4G MIMO-OFDM testbed which consists of high-speed configurations up to $4 \times 4 \mathrm{MIMO}$ and has flexible interfaces for digital and analog baseband, intermediate frequency (IF) and radio frequency (RF) connections. The FPGA implementations of the detectors are synthesized for a Xilinx Virtex-II $\mathrm{XC} 2 \mathrm{~V} 6000$ chip and they are designed to operate with a 66 $\mathrm{MHz}$ clock frequency which is the internal frequency used in the EB4G. The EB4G main technical parameters are listed in Table I.

The high level architecture of the LMMSE detector is presented in Figure 2. The LMMSE coefficient calculation block receives the scaled channel estimates $\mathbf{H}_{p}$ and $\frac{E_{s}}{N_{0}}$ as an input and gives the detector coefficient matrices $\mathbf{W}_{p}$ as an output for each subcarrier $p$. Adaptive scaling is applied to the LMMSE input values to set the values at optimal level in respect to the coefficient calculation block internal accuracy. The scaling is done separately for each subcarrier according to the largest channel estimate coefficient. The scaling is compensated for after the detection. The coefficient calculation operates at frame interval, i.e., they are recalculated every 80 


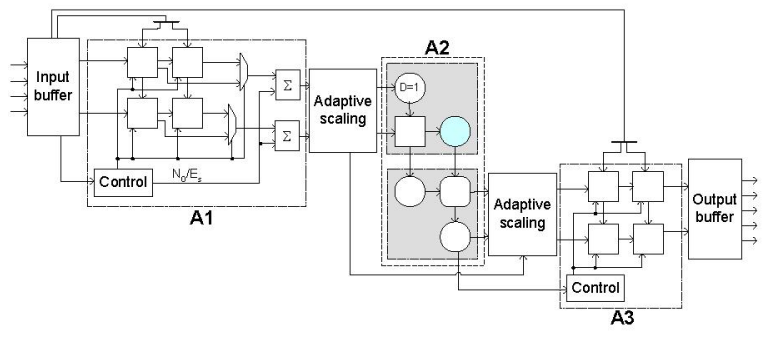

Fig. 4. The SGR based LMMSE detector architecture for $2 \times 2$ system.

TABLE I

EB4G MAIN PARAMETERS.

\begin{tabular}{|c|l|}
\hline Central frequency with RF units & $2400 \ldots 2500 \mathrm{MHz}$ \\
\hline Sample rate & $20 \mathrm{MHz}$ \\
\hline Total signal bandwidth & $16.9 \mathrm{MHz}$ \\
\hline Sub-carriers per OFDM symbol & 64 \\
\hline Data carriers per OFDM symbol & 52 \\
\hline OFDM symbol duration & $3.2 \mu \mathrm{s}$ \\
\hline Burst duration (CP + OFDM sym.) & $4 \mu \mathrm{s}$ \\
\hline Frame length & $80 \mu \mathrm{s}$ \\
\hline Data symbols in one frame & 16 \\
\hline Convolutional code & $\mathrm{CC}(4,2,7),[171,133]$ polynomial \\
\hline Convolutional decoder & Viterbi decoder \\
\hline Code rate & $1 / 2$ \\
\hline Supported modulations & BPSK, QPSK, 16QAM \\
\hline Channel estimator & LS - based estimator \\
\hline &
\end{tabular}

$\mu s$. The calculated $\mathbf{W}_{p}$ are stored to block random access memory (RAM). The received data $\mathbf{r}_{p}$ is buffered to first-in first-out (FIFO) memory before the data detection. The data detection, i.e., a matrix-vector multiplication, is done for all 52 subcarriers in burst interval $(4 \mu s)$.

The most complex part of the detector, the calculation of the LMMSE detector coefficients $\mathbf{W}_{p}$, is implemented using two different solutions, namely the CORDIC algorithm and SGR algorithm based solutions [12]. Other parts of the two considered detectors have been implemented using similar parts.

The CORDIC based implementation uses 16 bit fixed-point internal word lengths in coefficient matrix calculation, which includes the matrix-matrix multiplications, the CORDIC based $\mathrm{QRD}$ and the back substitution. The CORDIC algorithm that is used to calculate QRD is implemented with $i_{\max }=10$ iterations. It should be noted that the number of iterations and word length may be decreased depending on the required accuracy. A design with $i_{\max }=7$ iterations and 12 bit internal word length would require approximately $30 \%$ less slices in synthesis. The latency for calculating all $522 \times 2$

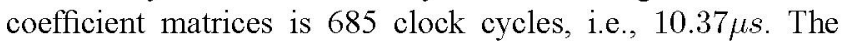
device utilization of CORDIC based LMMSE detector in EB4G is listed in Table II.

TABLE II

CORDIC BASED DETECTOR, DEVICE UTILIZATION FOR XC2V6000.

\begin{tabular}{|l|c|c|c|}
\hline Resource & Coeff. calc. & Whole detector & Available \\
\hline CLB Slices & $11910(35.2 \%)$ & $15834(46.9 \%)$ & 33792 \\
\hline Block RAMs & $6(4.2 \%)$ & $20(13.9 \%)$ & 144 \\
\hline Block Multipliers & $20(13.9 \%)$ & $32(22.2 \%)$ & 144 \\
\hline
\end{tabular}

TABLE III

SGR BASED DETECTOR, DEVICE UTILIZATION FOR XC2V6000

\begin{tabular}{|l|c|c|c|}
\hline Resource & Coeff. calc. & Whole detector & Available \\
\hline CLB Slices & $7422(22.0 \%)$ & $11346(33.6 \%)$ & 33792 \\
\hline Block RAMs & $14(9.7 \%)$ & $28(19.4 \%)$ & 144 \\
\hline Block Multipliers & $77(53.5 \%)$ & $89(61.8 \%)$ & 144 \\
\hline
\end{tabular}

The SGR based implementation uses mainly 18 bit fixedpoint internal word lengths coefficient matrix calculation, which includes the matrix-matrix multiplications, the SGR based $\mathrm{QRD}$ and the triangular matrix inversion. Adaptive scaling is done in coefficient calculation before the matrix inversion due to the high dynamic range requirements of the $\mathrm{SGR}$ based QRD. The matrix to be inverted in A2 part is scaled to a desired level according to the highest value of each matrix. The scaling then compensated for after the matrix inversion as illustrated in Figure 4. It was noted that the reciprocal divider needed in the QRD and in the matrix inversion calculation is the most accuracy demanding point in the matrix inversion calculation. The implemented divider uses 20 bit fixed-point word length and also three step adaptive scaling to reduce the required dynamic range of the signal. The latency for calculating all $522 \times 2$ coefficient matrices with SGR based implementation is 574 clock cycles, i.e., $8.69 \mu$ s. The device utilization of SGR based LMMSE detector in EB4G is listed in Table III.

\section{Performance examples}

The performance measurements are done with a EB4G hardware testbed and a Propsim C8 MIMO channel emulator is used to create the four baseband channels in the $2 \times 2 \mathrm{MIMO}$ system in real-time. A photo of the measurement configuration is shown in Figure 5 and the EB4G main technical parameters are listed in Table I. The performances of both CORDIC and SGR based detector implementations are measured at baseband and they are compared to simulation results. The simulations have been done in Matlab with a floating point representation. The effect of the LS estimator used in the realtime measurements is applied to Matlab model by adding noise to the channel coefficients [13].

A convolutionally coded spatial multiplexing (SM) transmission with quadrature phase shift keying (QPSK) and with bit-interleaving is applied with LMMSE detector and Viterbi decoder at the receiver. The $1 / 2$ rate convolutional code is applied with [171,133] polynomial and the coding is done over one OFDM symbol interval. A least squares (LS) based channel estimator is used in the EB4G with two OFDM pilot symbols per frame [13]. A correct value of $\frac{E_{s}}{N_{0}}$ is used in the measurements with no estimation error. Three different non line-of-sight (NLOS) channel profiles from WINNER [14] channel model are used as a channel in the measurements: indoor small office (A1), typical urban micro cell (B1), and suburban macro cell (C1). The effect of channel correlation properties is measured by using different antenna separations. An antenna separation of $0.5 \lambda$, i.e., $1 / 2$ wavelength of the used transmission frequency, is used at the mobile station (MS). At the base station (BS), antenna separations of $0.5 \lambda, 4 \lambda$, and $10 \lambda$ 


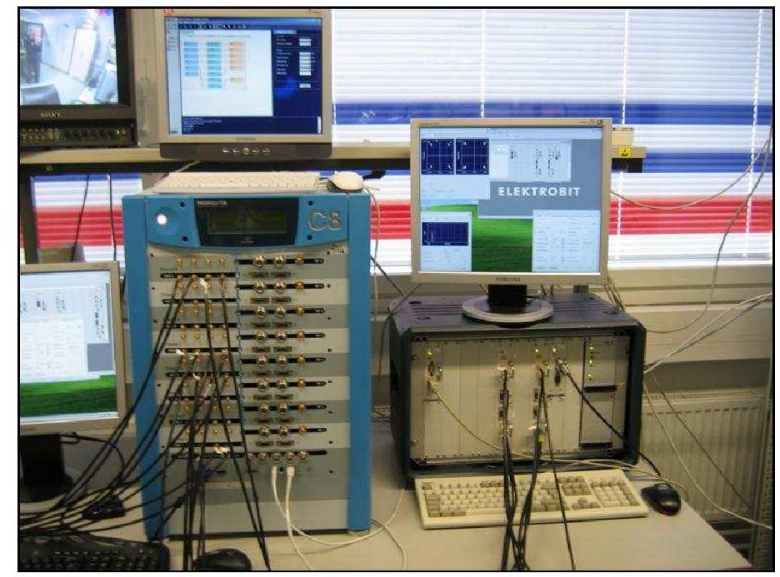

Fig. 5. The measurement configuration consisting of a Propsim C8 channel emulator on the left and a EB4G hardware testbed on the right.

are used. A lower value of antenna separation corresponds to more correlated antennas.

Bit error rate (BER) results with coded system in WINNER Al channel are shown in Figure 6. It can be noted that the total performance loss of the implemented system is between $2-2.5 \mathrm{~dB}$ at BER level of $10^{-2}$ with BS antenna separations of $4 \lambda$ and $10 \lambda$ compared to simulated results. The antenna separation of $0.5 \lambda$, i.e., the high correlation case, results in greater implementation loss. The channel realization with higher correlation results in higher eigenvalue spread which leads to higher dynamic range in the signal representation in the calculation of $\mathbf{W}_{p}$. The WINNER A1 channel scenario is rather flat fading and, in the case of a bad channel realization, it affects multiple subcarriers. Thus, the channel coding and the interleaving are not often capable to correct the errors. It can be noted that the BER performance of the SGR based detector starts to saturate earlier than the performance of the CORDIC based detector. This is due to the high dynamic range requirements of the SGR algorithm.

BER results with coded system in WINNER Bl channel are shown in Figure 7. In this case the performance loss between measurement and simulation results is between $1.5-2 \mathrm{~dB}$ at BER level of $10^{-2}$ for $4 \lambda$ and $10 \lambda$ antenna separations. The performance loss with $0.5 \lambda$ separation is now lower compared to WINNER A1 channel. In this case the WINNER B1 channel is more frequency selective, and, thus, the channel coding and the interleaving are able to decrease the effect of bad channel realizations. Also the performance gap between floating point simulations and fixed-point implementation is smaller as the occasional errors due to implementation are being corrected more effectively. Raw BER results in WINNER B1 channel are shown in Figure 8. It can be noted that the performance difference in simulation and measurement results is a bit larger in raw BER and the channel coding and the interleaving compensate for some of the errors.

BER results with coded system in WINNER $\mathrm{C} 1$ channel are shown in Figure 9. The performance loss due to higher antenna

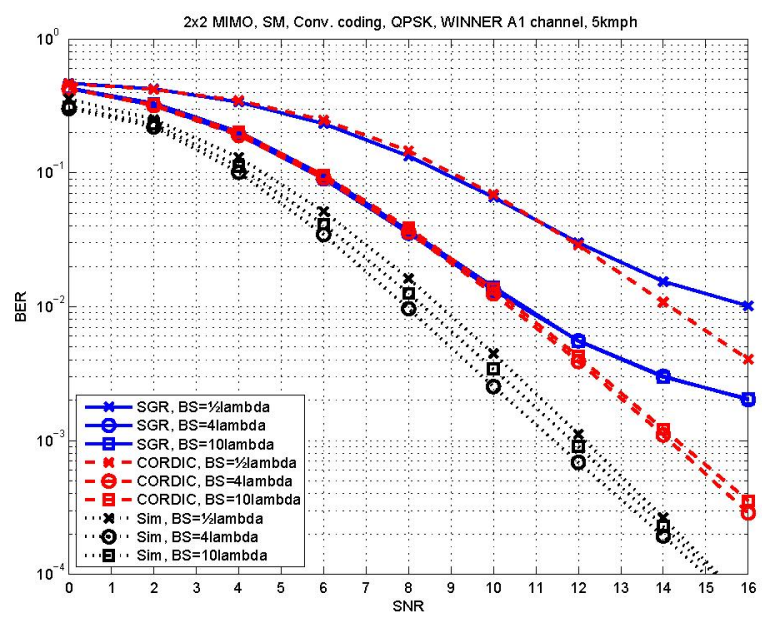

Fig. 6. Convolutional coded SM system with LMMSE detector and Viterbi decoder in WINNER A1 channel with $5 \mathrm{kmph}$ velocity.

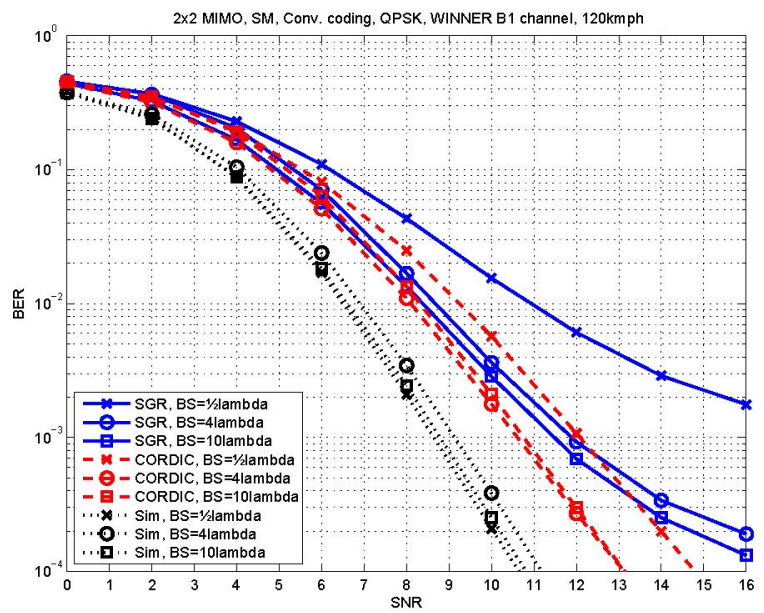

Fig. 7. Convolutional coded SM system with LMMSE detector and Viterbi decoder in WINNER B1 channel with $120 \mathrm{kmph}$ velocity.

correlation is higher in $\mathrm{C} 1$ channel compared to $\mathrm{A} 1$ and $\mathrm{B} 1$ channels. The effect of antenna spreading is larger at outdoor environments. However, the performances of simulation and measurement results are closer to each other. This is due that the dynamic range of the signal is lowest in WINNER C1 channel, and, thus, the performance loss is negligible.

The performance of the SM transmission with the LMMSE detector implementations is also compared to Alamouti [15] space-time block code (STBC) and single-input single-output (SISO) transmissions. The performance results for the same rate transmissions in WINNER Bl channel are shown in Figure 10. It can be seen that the SM and Alamouti schemes performance is quite similar in Bl channel and the gap to SISO transmission is clear.

\section{Summary AND Conclusions}

The performances of two FPGA implemented LMMSE based detectors were studied in EB4G MIMO-OFDM based hardware testbed in real-time radio channel. The detectors 


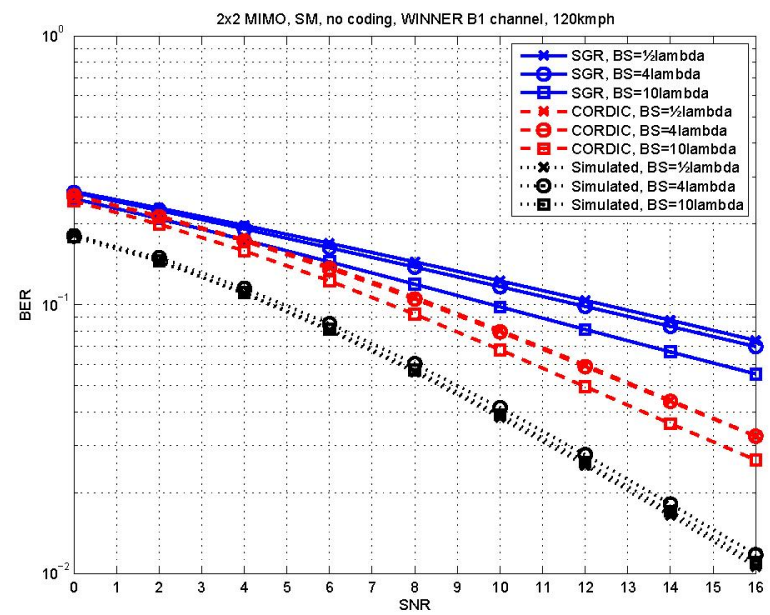

Fig. 8. Uncoded system with LMMSE detector in WINNER B1 channel with $120 \mathrm{kmph}$ velocity.

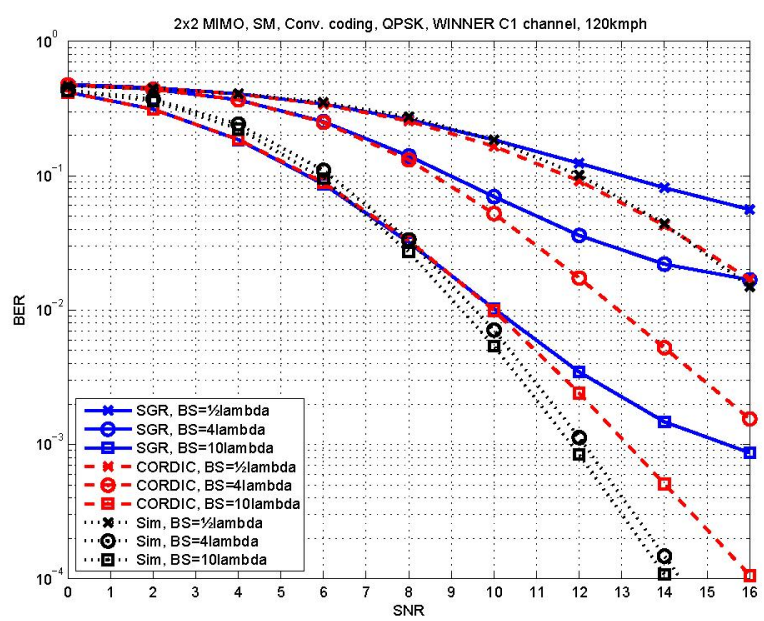

Fig. 9. Convolutional coded SM system with LMMSE detector and Viterbi decoder in WINNER C1 channel with $120 \mathrm{kmph}$ velocity.

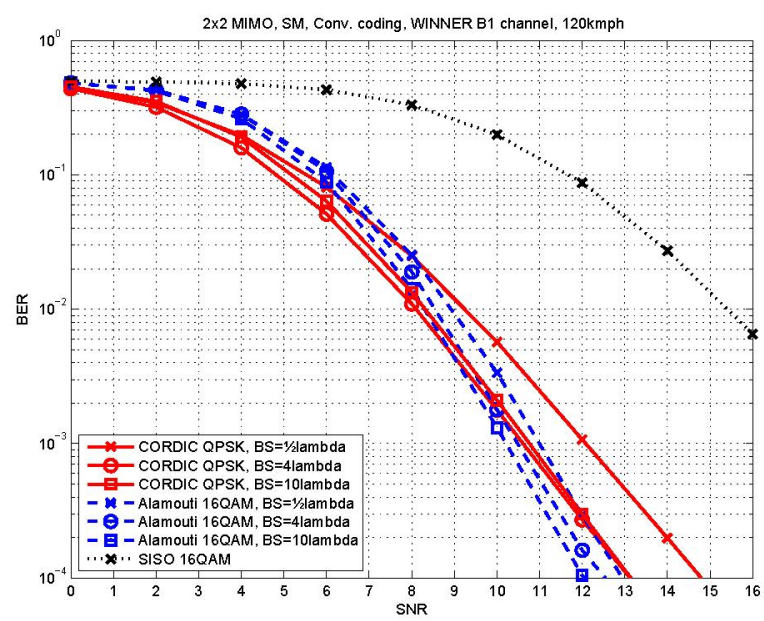

Fig. 10. Convolutional coded SM system with LMMSE detector and Viterbi decoder and Alamouti system in WINNER B1 channel with $120 \mathrm{kmph}$ velocity. were based on CORDIC and SGR algorithms, and designed using systolic array architectures and fixed-point arithmetic.

The measurement results were compared to Matlab floating point simulations. It was noted that the performance of the detectors was highly dependent on the channel scenario and the correlation properties of the channel. Typically the performance loss between simulated and measured results was between $1-2 \mathrm{~dB}$. The results showed that the SGR based detector implementation has problems with fixed-point arithmetic related to large dynamic range required in the signal representation. The problems would decrease with floating point arithmetic. The traditional CORDIC design seemed more suitable for considered system with fixed-point arithmetic. However, it was shown that both implementations work in real channel environment.

\section{ACKNOWLEDGEMENTS}

This work was done as a part of the MITSE project which was supported by Elektrobit, Nokia Networks, Nokia Technology Platforms, Texas Instruments, and the Finnish Funding Agency for Technology and Innovation, Tekes. The first author would also like to thank the Nokia foundation, and Jenny and Antti Wihuri foundation for supporting the research.

\section{REFERENCES}

[1] H. Bölcskei, D. Gesbert, and A. J. Paulraj, "On the capacity of OFDM based spatial multiplexing systems," IEEE Trans. Commun., vol. 50, no. 2, pp. 225-234, Feb., 2002.

[2] H. Yang, "A road to future broadband wireless access: MIMO-OFDMbased air interface," Communications Magazine, IEEE, vol. 43, no. 1, pp. 53-60, Jan., 2005.

[3] G.J. Foschini, "Layered space-time architecture for wireless communication in a fading environment when using multi-element antennas," pp. 41-59, Aug. 1996.

[4] G. H. Golub and C. F. Van Loan, Matrix Computations, 3rd ed., The Johns Hopkins University Press, Baltimore, 1996.

[5] S. Haykin, Adaptive Filter Theory, Prentice Hall, Englewood Cliffs, NJ, USA, 2nd edition, 1991.

[6] J.E. Volder, "The CORDIC Trigonometric Computing Technique," IRE Trans. on Electronic Computers, vol. EC-8, no. 3, pp. 330-4, 1959.

[7] R. Döhler, "Squared Givens Rotation," IMA Journal of Numerical Analysis, vol. 11, pp. 1-5, 1991.

[8] A. El-Amawy amd K.R. Dharmarajan, "Parallel VLSI algorithm for stable inversion of dense matrices," IEE Proceedings, vol. 136, 1989.

[9] W.M. Gentleman and H.T. Kung, "Matrix triangularization by systolic array," Proc. SPIE, Real-time signal processing IV, vol. 298, pp. 19-26, Bellingham, Washington, 1981.

[10] S.Y. Kung, VLSI Array Processors, Prentice-Hall, 1987.

[11] S. M. Kay, Fundamentals of Statistical Signal Processing: Estimation Theory, Prentice-Hall, Englewood Cliffs, NJ, USA, 1993.

[12] M. Myllylä, J-M Hintikka, M. Limingoja, A. Byman, J. Cavallaro, and M. Juntti, "Complexity Analysis of MMSE Detector Architectures for MIMO OFDM Systems," in In Proc. Asilomar Conference on Signals, Systems, and Computers, Pacific Grove, CA, USA, Oct. 30 - Nov. 2. 2005 , pp. $75-81$.

[13] H. Miao and M. Juntti, "Space-time channel estimation and performance analysis for wireless MIMO-OFDM systems with spatial correlation," IEEE Trans. Veh. Technol., vol. 54, no. 6, pp. 2003-2016, Nov., 2005.

[14] J. Salo, G. Del Galdo, J. Salmi, P. Kyösti, L. Hentilä, M. Milojevic, D. Laselva, P. Zetterberg, and C. Schneider, "MATLAB Implementation of the WINNER Phase I Channel Model," [Online], Dec. 2005, https:/www.ist-winner.org/ChannelModelImpl.htm.

[15] S. Alamouti, "A simple transmit diversity technique for wireless communications," IEEE J. Select. Areas Commun., vol. 16, no. 8, pp. 1451-1458, Oct 1998. 